\title{
Sensitivity to Non-Standard Interactions (NSI) with KM3NeT-ORCA
}

\section{The KM3NeT Collaboration ${ }^{* *}$}

\$ https://www.km3net.org/km3net-author-list-for-icrc-2019

\begin{abstract}
ORCA (Oscillations Research with Cosmics in the Abyss) is the low-energy detector of KM3NeT, the next generation underwater Cherenkov neutrino detector in the Mediterranean Sea. Although its primary goal is to determine the Neutrino Mass Ordering (NMO) with atmospheric neutrinos, its access to a wide range of energies and baselines makes it optimal to discover exotic physics beyond the Standard Model (SM) such as Non-Standard Interactions (NSI).

Non-Standard Interactions with neutrinos will introduce modifications in the SM potential, which will alter the event spectra at the ORCA detector. Using these NSI induced distortions in the flavour content over three flavour standard oscillations, it is possible to constrain NSI model parameters. This report outlines the sensitivity that ORCA can achieve in putting upper bounds on some of these non-standard neutrino interaction parameters.
\end{abstract}

Corresponding authors: N. R. Khan Chowdhury ${ }^{1}$, T. Thakore ${ }^{1}$, J. A. B. Coelho ${ }^{2}$, J. D. Zornoza $^{1}$ and S. Navas ${ }^{3}$

${ }^{1}$ IFIC - Instituto de Física Corpuscular (Univ. de Valéncia - CSIC), E-46980 Paterna, Spain

${ }^{2}$ LAL, Univ. Paris-Sud, CNRS / IN2P3, Paris-Saclay University, 91440 Orsay, France

${ }^{3}$ Dpto. de Física Teórica y del Cosmos \& C.A.F.P.E, University of Granada, 18071 Granada, Spain

Email: nafis.chowdhuryeific.uv.es, tarak.thaoredific.uv.es, coelho@lal.in2p3.fr, zornoza@ific.uv.es, navas@ugr.es

36th International Cosmic Ray Conference -ICRC2019-

July 24th - August 1st, 2019

Madison, WI, U.S.A.

${ }^{*}$ for collaboration list see PoS(ICRC2019)1177 


\section{Introduction}

The phenomena of Neutrino Oscillations has been well established by a gamut of experiments over the last two decades. Theories beyond the Standard Model (SM) introduce heavy, TeV-scale bosons which can produce additional interactions apart from the SM MSW matter effects. Such nonstandard neutrino interactions are expected to produce a significant departure from the prediction for standard 3 flavour neutrino oscillations. In this contribution, we explore the impact of the neutral current non-standard interactions of neutrinos with matter fermions while traversing through long distances across the Earth matter.

\section{Oscillation framework in presence of Non-Standard Interactions}

Non-Standard Interactions (NSI) of neutrinos can be modelled as perturbations in the standard neutrino propagation Hamiltonian [1]. In this analysis, we consider non-standard interactions between neutrinos and $d$-quarks present in Earth matter unless otherwise mentioned. The full three-flavour Hamiltonian governing the propagation of neutrinos in the presence of NSI is

$$
H=\frac{1}{2 E} U\left[\begin{array}{ccc}
0 & 0 & 0 \\
0 & \Delta m_{21}^{2} & 0 \\
0 & 0 & \Delta m_{31}^{2}
\end{array}\right] U^{\dagger}+2 \sqrt{2} G_{F} N_{e}(x)\left[\begin{array}{ccc}
1+\varepsilon_{e e} & \varepsilon_{e \mu} & \varepsilon_{e \tau} \\
\varepsilon_{e \mu}^{*} & \varepsilon_{\mu \mu} & \varepsilon_{\mu \tau} \\
\varepsilon_{e \tau}^{*} & \varepsilon_{\mu \tau}^{*} & \varepsilon_{\tau \tau}
\end{array}\right]
$$

where, $E$ is the neutrino energy, $U$ is the PMNS matrix, which describes the standard neutrino mixing as rotations between the flavour and mass eigenstates parametrized by unique mixing angles $\theta_{i j}$ and $\Delta m_{i j}^{2}$ is the mass-squared difference between two mass eigenstates viz. $i$ and $j . G_{F}$ is the Fermi coupling constant, $N_{e}(x)$ is the electron number density along the neutrino path, and the $\varepsilon_{\alpha \beta}$ represent the NSI coupling parameters. The NSI phases are assumed to be at zero in this analysis.

Figure 1 shows the effect of the presence of non-standard interactions in the two most dominant channels at ORCA. The effect for neutrinos in the Nornal Ordering (NO) scheme is the same as for anti-neutrinos in the Inverted Ordering (IO) scheme.
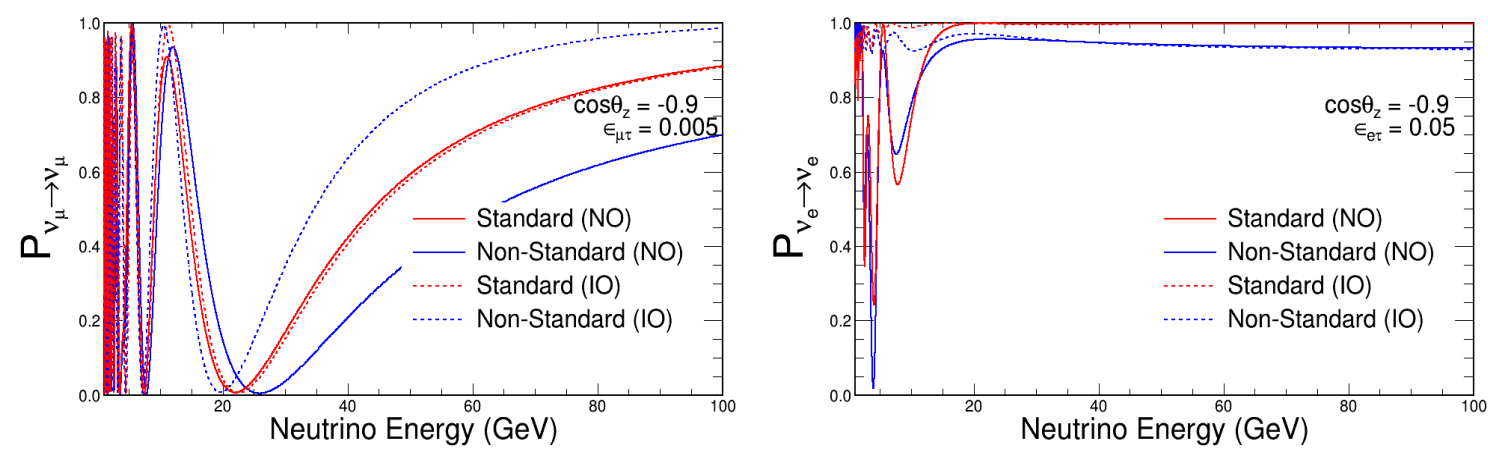

Figure 1: Oscillation probabilities in the $v_{\mu} \rightarrow v_{\mu}$ (left) and $v_{e} \rightarrow v_{e}$ (right) channel as a function of neutrino energy for a fixed value of zenith angle $\left(\theta_{z}\right)$. The solid (dashed) curves are for NO (IO). The values of NSI parameters for which the blue curves are drawn are quoted. 


\section{The KM3NeT-ORCA detector}

The KM3NeT-ORCA detector [2], currently being installed at a depth of $2450 \mathrm{~m}$ in the Mediterranean Sea, is a megaton-scale water Cherenkov detector located $40 \mathrm{~km}$ offshore Toulon, France. Upon completion, the detector will consist of 115 detection units (DUs), each of which will comprise 18 spherical, 17" diameter Digital Optical Modules (DOMs) housing 31 3" PMTs and associated electronics. The vertical spacing between the DOMs is $9 \mathrm{~m}$ and the horizontal spacing between the DUs is $23 \mathrm{~m}$, amounting to a total instrumented volume of $\sim 8$ Mton. The granularity of the detector layout makes it optimal to detect neutrinos with energies as low as $3 \mathrm{GeV}$.

\section{4. $\chi^{2}$ maps at the detector level}

Depending on the Cherenkov signatures of the outgoing lepton from the $v_{e}$ and $v_{\mu}$ charged current (CC) and neutral current (NC) interactions, two distinct event topolgies are observed at the detector: track-like and shower-like events. While $v_{\mu} \mathrm{CC}$ interactions mostly account for track-like topology, shower-like topology has events from both $v_{e}$ charged current (CC) and neutral current (NC) interactions of all flavours.

Figure 2 shows the signed- $\chi^{2}$ maps for reconstructed events in the track-like and shower-like event topologies for 1 year of full ORCA (115 DUs) runtime. 20 logarithmic bins were chosen in reconstructed neutrino energy $(E)$ between 3 and $100 \mathrm{GeV}$, and 20 linear bins in cosine of the reconstructed zenith angle $\left(\theta_{z}\right)$ between -1 and 0 . The absolute value of $\chi^{2}$ is stored, so that each bin content represents the contribution to the total $\chi^{2}$, but retaining the sign information to represent the excess and deficits. The statistical $\chi^{2}$ for each bin is computed from

$$
\chi_{E, \theta_{z}}^{2}\left(\varepsilon_{\alpha \beta}\right)=\frac{\left(N_{E, \theta_{z}}^{\text {model }}\left(\varepsilon_{\alpha \beta}\right)-N_{E, \theta_{z}}^{\text {data }}\left(\varepsilon_{\alpha \beta}=0\right)\right) \times\left|N_{E, \theta_{z}}^{\text {model }}\left(\varepsilon_{\alpha \beta}\right)-N_{E, \theta_{z}}^{\text {data }}\left(\varepsilon_{\alpha \beta}=0\right)\right|}{N_{E, \theta_{z}}^{\text {data }}\left(\varepsilon_{\alpha \beta}=0\right)},
$$

where the superscipt model represents the NSI case with $\left|\varepsilon_{e \tau}\right|=0.05$. All other NSI parameters are fixed at zero.
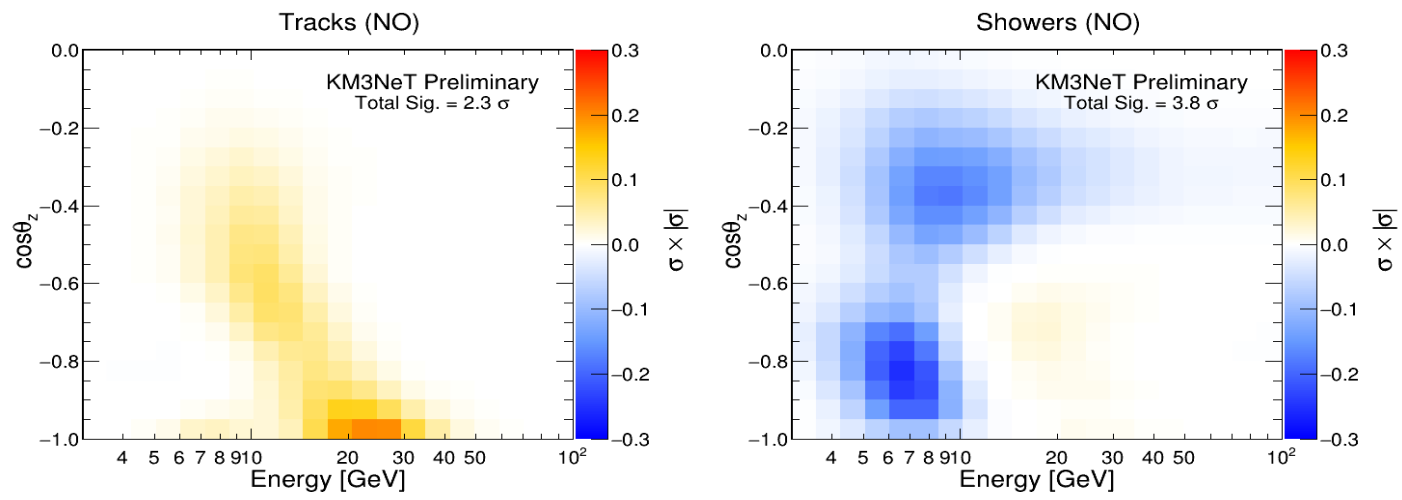

Figure 2: Statistical $\chi^{2}$ as a function of reconstructed neutrino energy $(E)$ and direction $\left(\cos \theta_{z}\right)$ for tracklike (left) and shower-like (right) event topologies. The NSI model is represented by setting $\left|\varepsilon_{e \tau}\right|=0.05$ and fixing all other $\varepsilon_{\alpha \beta}$ at 0 . Normal Ordering is assumed in both cases. The color code indicates the significance times its absolute value as defined in Equation 4.1. The total sensitivity quoted is the sum of the absolute value of the statistical $\chi^{2}$ for each bin. 


\section{Numerical Analysis}

In our analysis, we use the following test statistic, that gives us the median sensitivity of the experiment to NSI parameters.

$$
\begin{aligned}
\chi_{S}^{2}\left(\varepsilon_{\alpha \beta}\right)=2 \sum_{E, \theta_{z}}\left(N_{E, \theta_{z}}^{\text {model }}\left(\varepsilon_{\alpha \beta}\right)\left(1+\sum_{k} f_{E, \theta_{z}}^{k} \zeta_{k}\right)-N_{E, \theta_{z}}^{\text {data }}\left(\varepsilon_{\alpha \beta}=0\right)\right. \\
\left.+N_{E, \theta}^{\text {data }}\left(\varepsilon_{\alpha \beta}=0\right) \ln \frac{N_{E, \theta_{z}}^{\text {data }}\left(\varepsilon_{\alpha \beta}=0\right)}{N_{E, \theta_{z}}^{\text {model }}\left(\varepsilon_{\alpha \beta}\right)\left(1+\sum_{k} f_{E, \theta_{z}}^{k} \zeta_{k}\right)}\right)+\sum_{k} \zeta_{k}^{2} .
\end{aligned}
$$

$E$ and $\theta_{z}$ are the physical obervables in the Poissonian $\chi_{S}^{2}$ defined above. $N_{E, \theta_{z}}^{\text {model }}$ and $N_{E, \theta_{z}}^{\text {data }}$ denote the expected and observed number of track/shower events in a given $\left[E, \theta_{z}\right]$ bin. Systematics are included in our simulation using the "pull" method $[3,4]$. In the above equation, $\zeta_{k}$ is the fitting parameter for the $k$-th systematic error and $f^{k}$ is the fractional change of the event rate in the $(E, \theta)$ bin due to a $\sigma_{k}$ change in the $k$-th systematic error. The additional term $\sum_{k} \zeta_{k}^{2}$ is the penalty imposed for moving the $k$-th systematic error away from its standard value by $\sigma_{k} \zeta_{k}$.

The individual contributions from track-like and shower-like events are added in quadrature to compute the total significance.

$$
\chi_{S}^{2}=\chi_{\text {tracks }}^{2}+\chi_{\text {showers }}^{2}
$$

Table 1 lists nuisance parameters over which marginalisation has been done to minimise the value of $\chi_{S}^{2}\left(\varepsilon_{\alpha \beta}\right)$. Gaussian priors similar to the mass ordering analysis are included for the flux systematics. No priors are used on the atmospheric oscillation parameters since they are also supposed to be measured by ORCA and the starting value of $\theta_{23}$ is always chosen in the lower octant. Solar parameters are kept fixed at their nominal values. The mixing angle $\theta_{13}$ has a large effect on oscillation probabilities. Hence existing experimental constraints are necessary to exploit the full potential of ORCA for measuring NMO and the NSI parameters. NSI parameters, except the ones for which the sensitivity is estimated, are fixed at zero.

\section{Results}

ORCA sensitivities are generated assuming either Normal or Inverted Ordering for the neutrino mass hierarchy and 3 flavour oscillations with the set of true parameters adopted from NuFit 3.2 [5]. The sensitivities are calculated after fitting over the nuisance parameters and marginalising over the oscillation parameters as listed in Table 1.

In Figure 3, the sensitivities to the individual parameters, $\varepsilon_{e \mu}, \varepsilon_{e \tau}, \varepsilon_{\mu \tau}$ and $\varepsilon_{\tau \tau}$ are shown. Sensitivity lines are drawn assuming either NO (red) or IO (blue). 


\begin{tabular}{lccc}
\hline parameters & treatment & true values & $\sigma_{k}$ \\
\hline$\Delta m_{21}^{2} / 10^{-5} \mathrm{eV}^{2}$ & fix & 7.40 & - \\
$\Delta m_{31}^{2} / 10^{-3} \mathrm{eV}^{2}$ & fitted & 2.494 & free \\
$\theta_{12}\left(^{\circ}\right)$ & fix & 33.62 & - \\
$\theta_{13}\left({ }^{\circ}\right)$ & fitted & 8.54 & 0.15 \\
$\theta_{23}\left({ }^{\circ}\right)$ & fitted & 47.2 & free \\
$\delta_{C P}\left({ }^{\circ}\right)$ & fitted & 234 & free \\
Flux norm. & fitted & 1 & $10 \%$ \\
$\mathrm{NC}$ scale & fitted & 1 & $5 \%$ \\
Energy scale & fitted & 1 & $3 \%$ \\
$v_{\mu} / \nu_{e}$ skew & fitted & 0 & $5 \%$ \\
$v / \bar{v}$ skew & fitted & 0 & $3 \%$ \\
\hline
\end{tabular}

Table 1: Full list of nuisance parameters along with their statistical treatment and priors (if any).
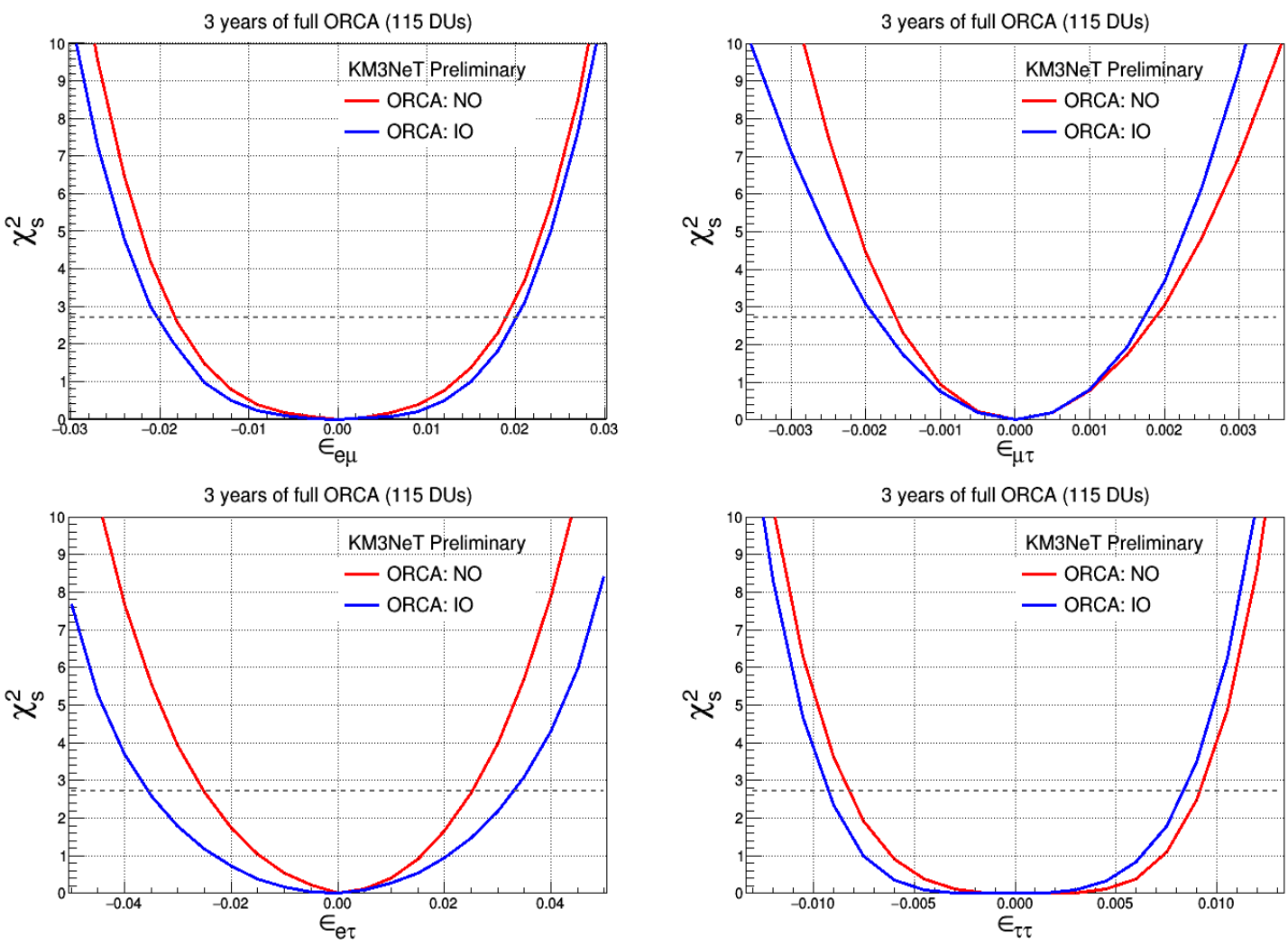

Figure 3: Sensitivities for ORCA towards different NSI parameters. For each of the plots, the NSI parameters except the one appearing on the plots are kept fixed at zero.

In Figure 4, the 90\% C.L. region in the NSI parameter space allowed after 3 years of running of ORCA is shown. These are obtained for the case, where the true values of the NSI parameters are assumed to be zero $(0,0)$. The contours are drawn after profiling over the nuisance parameters and marginalisation over the oscillation parameters. 

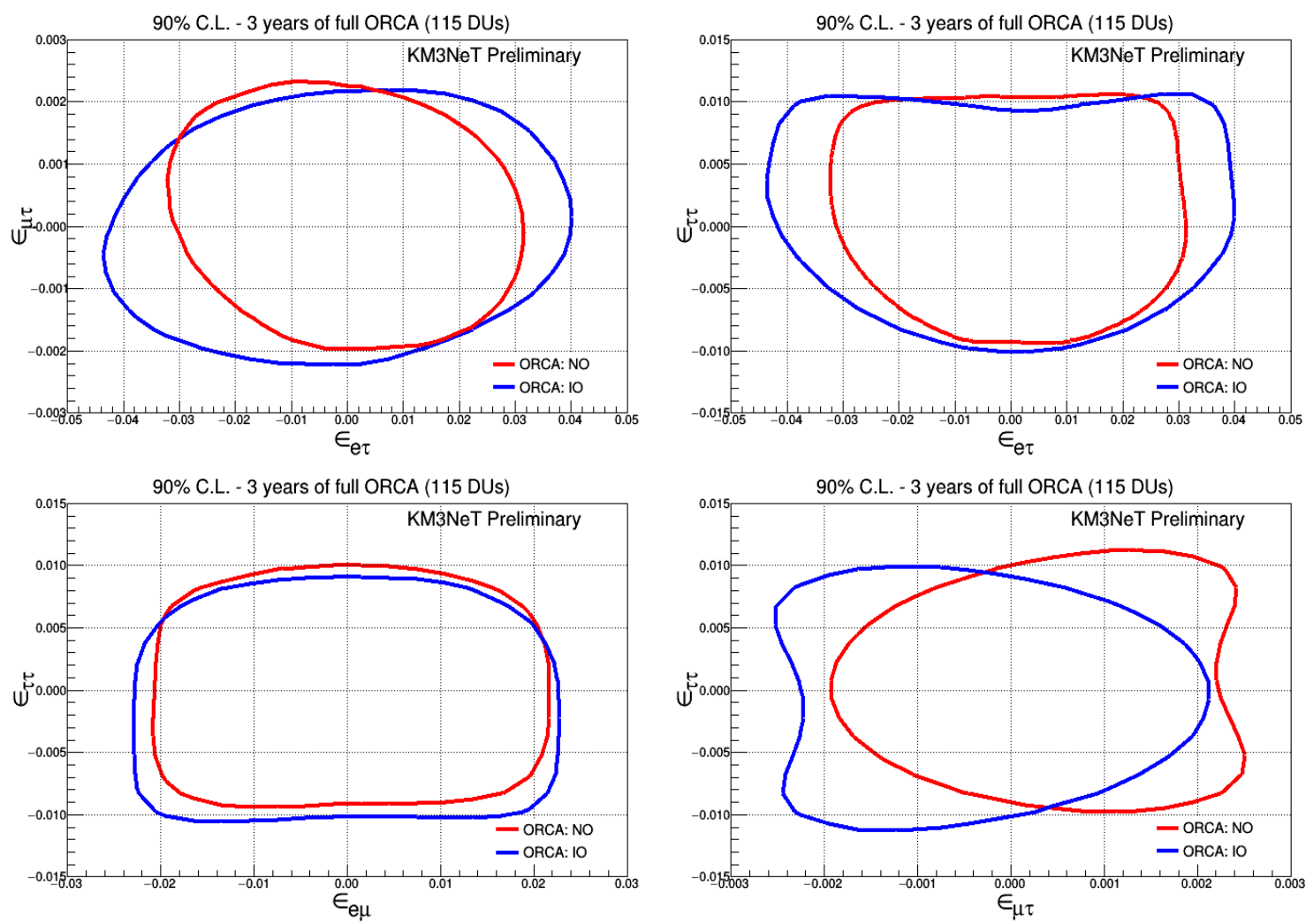

Figure 4: Correlated NSI parameter sensitivities for ORCA in different NSI phase spaces. For each of the plots, the NSI parameters not appearing on the plots are kept fixed at zero. The contours correspond to $90 \%$ CL as defined by $\chi^{2}=\chi_{\min }^{2}+4.61$. Data are generated for $\left(\varepsilon_{i j}, \varepsilon_{k l}\right)=(0,0)$.

\section{Hybrid model approximation and comparison with other experiments}

The ORCA NSI sensitivities, shown so far, are obtained in the 3 flavour oscillation framework as explained in Section 2. In this section, we assume 2 flavour oscillations with two different combinations of sets of NSI parameters for comparison with already existing limits from IceCube [6] and Super-K [7].

A model in which the NSI effects in the $v_{\mu}-v_{\tau}$ coexist with the standard two-flavour $\left(\theta_{12}, \theta_{13}\right.$, and $\left.\Delta m_{21}^{2}=0\right) v_{\mu} \leftrightarrow v_{\tau}$ neutrino oscillations is labelled as the two-flavour hybrid model. All NSI that couple to $v_{e}$ i.e, $\varepsilon_{e \alpha}$ are set to zero. The remaining non-zero parameters $\left(\varepsilon_{\mu \mu}, \varepsilon_{\mu \tau}\right.$ and $\left.\varepsilon_{\tau \tau}\right)$ introduces matter-dependent distortions to standard $v_{\mu} \leftrightarrow v_{\tau}$ oscillations and can be exploited to put limits on NSI parameters in the $\mu-\tau$ sector.

The red lines in either of the plots in Figure 5 shows the final results when marginalised over the nuisance parameters listed in Table 1. ORCA sensitivity lines are superimposed with contours from IceCube [6] and Super-K [7] for comparison. 

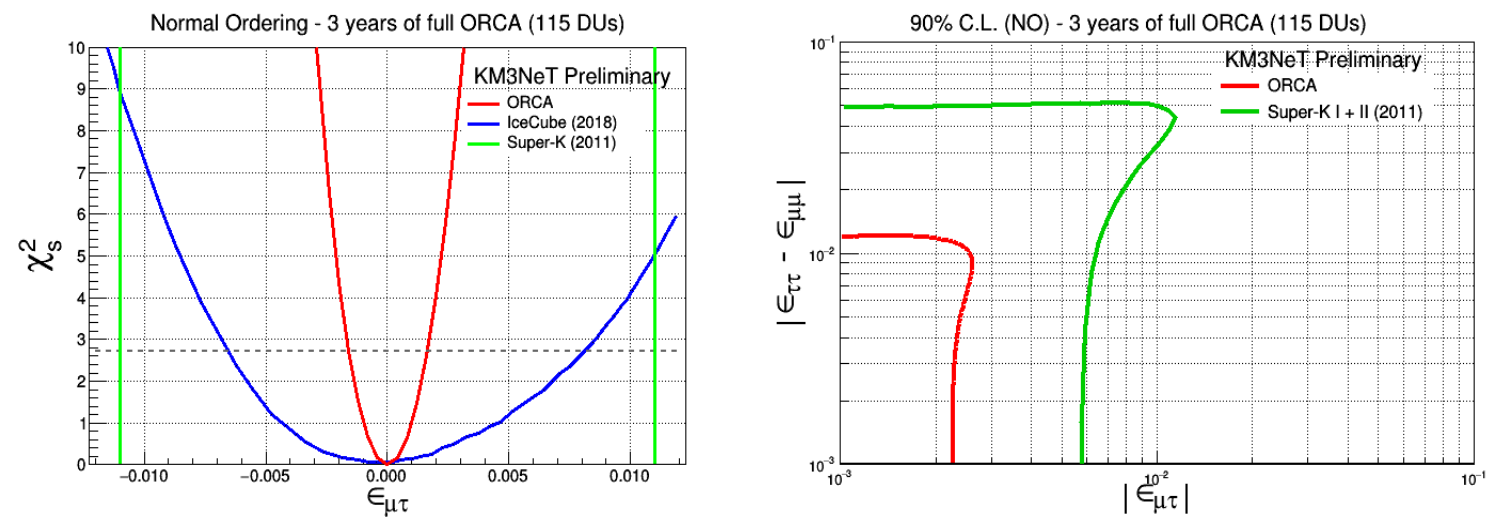

Figure 5: (On the left) Sensitivity to NSI parameter $\varepsilon_{\mu \tau}$ in the two-flavour hybrid model assuming $\varepsilon_{\mu \mu}=\varepsilon_{\tau \tau}$ $=0$; for ORCA (red), IceCube (blue) and Super-K (green). (On the right) Allowed NSI parameter region in the $\left|\varepsilon_{\mu \tau}\right|$ vs $\left|\varepsilon_{\tau \tau}-\varepsilon_{\mu \mu}\right|$ plane in the two-flavour hybrid model.

A model in which the NSI effects in the $v_{e}-v_{\tau}$ coexist with the standard two-flavour $\left(\theta_{12}, \theta_{13}\right.$, and $\left.\Delta m_{21}^{2}=0\right) v_{\mu} \leftrightarrow v_{\tau}$ neutrino oscillations and all $\varepsilon_{\mu \alpha}$ are set to zero is labelled as the threeflavour hybrid model. The analysis procedure is similar to that followed in the two-flavour hybrid model. A value of $\chi^{2}$ is calculated at each grid point in the two dimensional parameter space of $\varepsilon_{e} \tau$ and $\varepsilon_{\tau \tau}$ for a given value of $\varepsilon_{e e}(=0$, in our case). The exclusion region in this three-flavour hybrid model is shown in Figure 6.

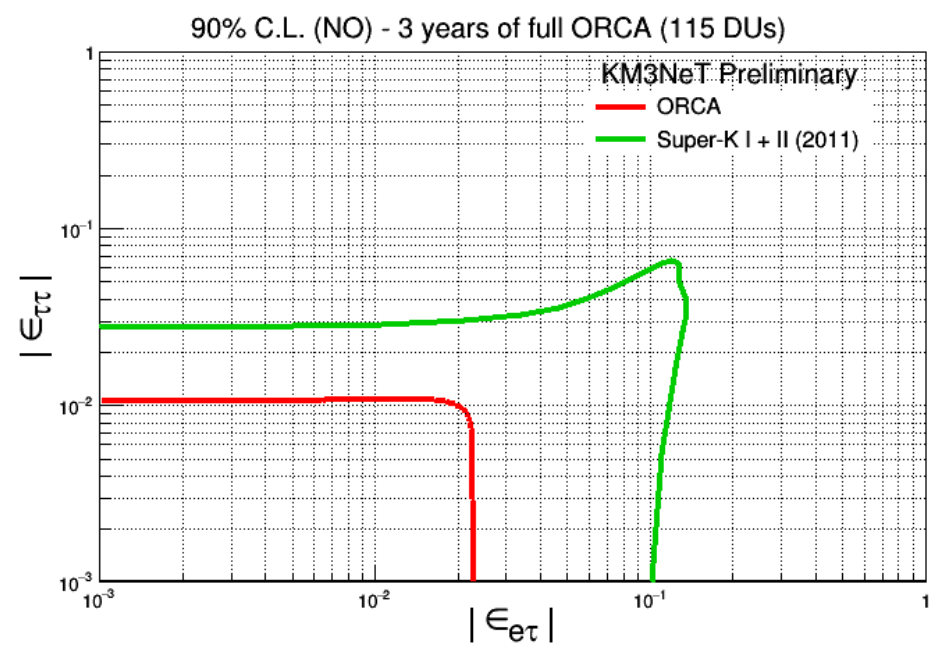

Figure 6: Allowed NSI parameter region in the $\left|\varepsilon_{e \tau}\right|$ vs $\left|\varepsilon_{\tau \tau}\right|$ plane assuming $\varepsilon_{e e}=0$. The ORCA contour is drawn for the three-flavour hybrid model analysis considering NSI $d$-quark-couplings.

\section{Summary}

In this contribution, the impact of non-standard interactions on the signal at KM3NeT-ORCA is probed and the discovery potential of ORCA towards different NSI parameters has been discussed. We give confidence level contours in the NSI parameter phase spaces that is expected to 
be allowed from 3 year of ORCA runtime. ORCA sensitivities are better than current limits from IceCube and Super-K by a factor of 4 almost.

\section{Acknowledgement}

We gratefully acknowledge the financial support of the Ministry of Science, Innovation and Universities: State Program of Generation of Knowledge, ref. PGC2018-096663-B-C41 (MCIU / FEDER), Spain.

\section{References}

[1] T. Ohlsson, Status of non-standard neutrino interactions, Rept. Prog. Phys. 76 (2013) 044201 [1209.2710].

[2] KM3NeT collaboration, Letter of intent for KM3NeT 2.0, J. Phys. G43 (2016) 084001 [1601.07459].

[3] R. Gandhi, P. Ghoshal, S. Goswami, P. Mehta, S. U. Sankar and S. Shalgar, Mass Hierarchy Determination via future Atmospheric Neutrino Detectors, Phys. Rev. D76 (2007) 073012 [0707.1723].

[4] A. Ghosh, T. Thakore and S. Choubey, Determining the Neutrino Mass Hierarchy with INO, T2K, NOvA and Reactor Experiments, JHEP 04 (2013) 009 [1212.1305].

[5] I. Esteban, M. C. Gonzalez-Garcia, M. Maltoni, I. Martinez-Soler and T. Schwetz, Updated fit to three neutrino mixing: exploring the accelerator-reactor complementarity, JHEP 01 (2017) 087 [1611.01514].

[6] ICECUBE collaboration, Search for Nonstandard Neutrino Interactions with IceCube DeepCore, Phys. Rev. D97 (2018) 072009 [1709.07079].

[7] SUPER-KAMIOKANDE collaboration, Study of Non-Standard Neutrino Interactions with Atmospheric Neutrino Data in Super-Kamiokande I and II, Phys. Rev. D84 (2011) 113008 [1109.1889]. 